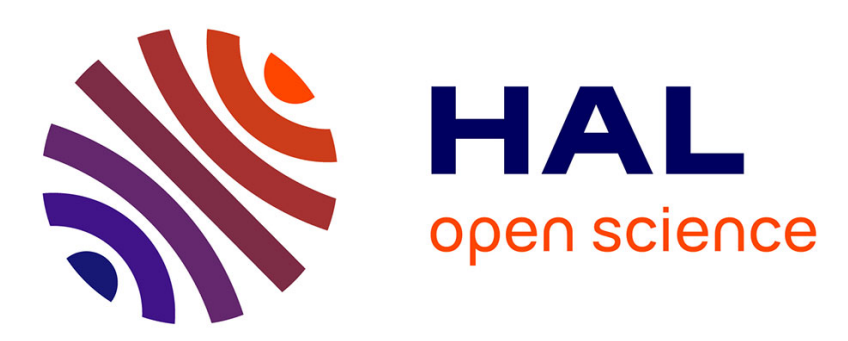

\title{
Numerical model to predict unsteady cavitating flow behaviour in inducer blade cascades
}

Regiane . Fortes Patella, Olivier Coutier-Delghosa, Jérôme Perrin, Jean-Luc

Reboud

\section{- To cite this version:}

Regiane . Fortes Patella, Olivier Coutier-Delghosa, Jérôme Perrin, Jean-Luc Reboud. Numerical model to predict unsteady cavitating flow behaviour in inducer blade cascades. Journal of Fluids Engineering, 2007, 129 (2), pp.128-135. 10.1115/1.2409320 . hal-00265997

\section{HAL Id: hal-00265997 https://hal.science/hal-00265997}

Submitted on 3 Jan 2020

HAL is a multi-disciplinary open access archive for the deposit and dissemination of scientific research documents, whether they are published or not. The documents may come from teaching and research institutions in France or abroad, or from public or private research centers.
L'archive ouverte pluridisciplinaire HAL, est destinée au dépôt et à la diffusion de documents scientifiques de niveau recherche, publiés ou non, émanant des établissements d'enseignement et de recherche français ou étrangers, des laboratoires publics ou privés.

\section{(c)(1)}

Distributed under a Creative Commons Attribution| 4.0 International License 


\section{Numerical Model to Predict Unsteady Cavitating Flow Behavior in Inducer Blade Cascades}

The cavitation behavior of a four-blade rocket engine turbopump inducer is simulated. A two-dimensional numerical model of unsteady cavitation was applied to a blade cascade drawn from an inducer geometry. The physical model is based on a homogeneous approach of cavitation, coupled with a barotropic state law for the liquid/vapor mixture. The numerical resolution uses a pressure-correction method derived from the SIMPLE algorithm and a finite volume discretization. Unsteady behavior of sheet cavities attached to the blade suction side depends on the flow rate and cavitation number. Two different unstable configurations of cavitation are identified. The mechanisms that are responsible for these unstable behaviors are discussed, and the stress fluctuations induced on the blade by cavitation instabilities are estimated.
R. Fortes-Patella ${ }^{1}$ e-mail: fortes@hmg.inpg.fr

0. Coutier-Delgosha ${ }^{2}$

J. Perrin

\author{
J. L. Reboud ${ }^{3}$ \\ Laboratoire des Ecoulements Géophysiques \\ et Industriels, \\ LEGI-INPG, \\ BP 53, 38041 Grenoble cedex 9, France
}

\section{Introduction}

To achieve operation at high rotational speed and low inlet pressure, rocket engine turbopumps are generally equipped with an axial inducer stage. Under such operating conditions, cavitation develops on the suction side of the blades and at inducer periphery near the tip. When pressure is decreased from cavitation inception, vapor develops more and more and, finally, leads to the inducer performance breakdown (Fig. 1).

Between low-cavitation conditions and the performance drop, for some particular range of the cavitation number, unsteady phenomena may appear, associated with different blade cavitation patterns. Figure 2 illustrates successive flow patterns observed experimentally in the case of a $\mathrm{H} 2$ inducer for different values of the cavitation parameter $\tau^{\prime}[1]$.

At cavitation inception, a steady and balanced flow pattern with one short attached cavity on each blade is observed from flow visualizations (Fig. 2(1)). When the cavitation parameter is slightly decreased, a steady and alternate cavitating configuration appears (only on four-blade inducers) with alternatively one short and one long cavity (Fig. 2(2)). Then flow visualizations achieve to identify an unsteady flow pattern (Fig. 2(3)): rotating cavitation appears at low cavitation parameter [2] just above breakdown. Unbalanced attached cavities are observed in the different channels, their distribution rotating faster than the inducer $[1,3]$ and leading to large radials loads on the shaft [4]. Finally, near the breakdown of the inducer, a steady and balanced flow pattern with fully developed cavitation is observed (Fig. 2(5)).

Over the last few years, numerical models have been developed to predict the cavitation instability in inducers [5]. These models are based on stability analyses and linear approach, taking into account the total flow-rate variations through a cavitating bladeto-blade channel [6-9], or calculating the flow around attached cavities [10-12].

To improve the understanding and the prediction capability of cavitation instabilities, numerical and experimental analyses are developed through collaborations between the LEGI, the Rocket

\footnotetext{
${ }^{1}$ Corresponding author.

${ }^{2}$ Now at LML/ENSAM Lille.

${ }^{3}$ Now at CNRS-LEMD, University of Grenoble, France.

Contributed by the Fluids Engineering Division of ASME for publication in the Journal of Fluids EngINEERING. Manuscript received November 25, 2003; final manuscript received May 23, 2006. Review conducted by Joseph Katz.
}

Engine Division of Snecma and the French space agency CNES. In the present paper, the cavitation behavior of a four-blade inducer is simulated using a two-dimensional (2D) model of unsteady cavitating flow developed at LEGI [13-16].

The paper illustrates a qualitative study whose aims are better understanding the instability phenomena and to propose a methodology for further parametric study. In spite of the progress of CFD, 3D unsteady calculations in inducer geometries are very time consuming and 2D approach is still interesting (even if it cannot represent completely the original three-dimensional case).

\section{Physical and Numerical Model}

Numerical simulation of the cavitating flow in the inducer was performed with the objective to take into account the cavitation sheets attached to the blades and their unsteady behavior. The 2D numerical model of unsteady cavitating flow IZ, developed in previous studies with the support of the CNES-Centre National d'Etudes Spatiales [13-15], was adapted to 2D blade cascades [16]. The main features of the models are:

- The liquid-vapor mixture is described as a single fluid, whose density $\rho$ rapidly varies between the pure liquid density $\rho_{1}$ and the pure vapor one $\rho_{\mathrm{v}}$ when the static pressure in the flow field reaches the vapor pressure. The fluid density is managed by a barotropic state law $\rho(\mathrm{p})$, as described in previous works. The barotropic law is given as a function of the maximum slope $1 / A_{\min }^{2}$, where $A_{\min }^{2}=\partial P / \partial \rho$.

- 2D unsteady Reynolds-averaged Navier-Stokes equations are applied to model the homogeneous mixture. The numerical resolution uses a pressure-correction method derived from the SIMPLE algorithm on 2D structured curvilinearorthogonal meshes. It is based on a conservative finite volume formulation (HLPA nonoscillatory second-order convection scheme) and on an implicit method for the time discretization. The used turbulence model is the classical $k-\varepsilon$ RNG model, associated with wall functions along solid boundaries.

- Other boundary conditions are (Fig. 5): imposed velocity at the mesh inlet (the flow velocity is deduced from the classical value $\left.Q / \rho S_{\text {flow }}\right)$, imposed static pressure at the outlet, and periodicity or connection conditions between the different channels of the blade cascade. These conditions are ap- 


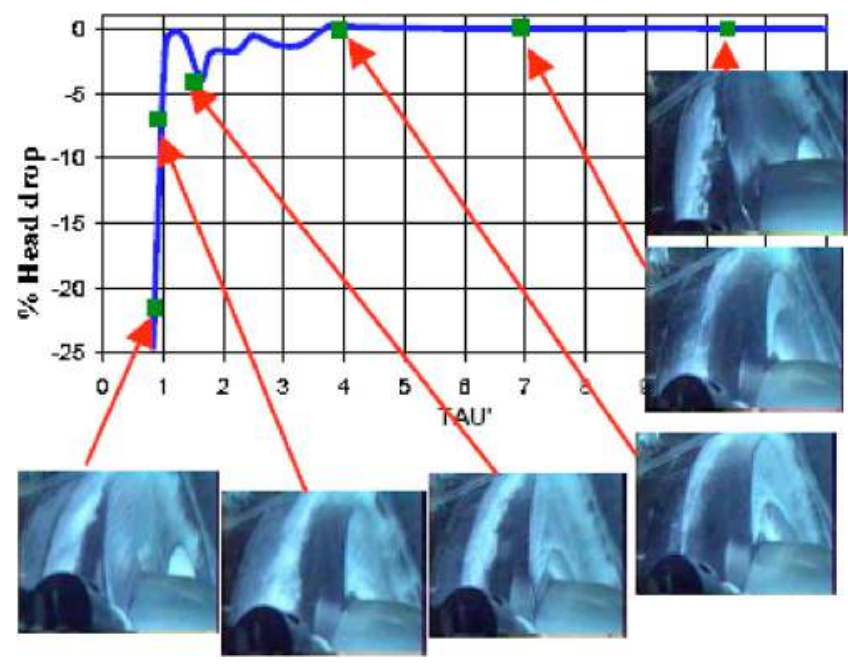

Fig. 1 Experimental performance chart for a H2 turbopump inducer (tests in cold water) [1]

plied along non-matching boundaries, through a special treatment developed in [16] to assure the best transfer of both mass and momentum fluxes.

The details of the numerical resolution were presented by [15] and [16]. A complete validation of the method was performed, and the influence of the numerical parameters was widely investigated. The results are reported in [15].

\section{Geometry}

The three-dimensional (3D) inducer geometry considered in the present study is illustrated by the Fig. 3. Even at nominal flowrate, the inducer is designed to operate with nonzero incidence angle. Nevertheless, in the range of running conditions, the angle of attack remains very small because of the variable thread and

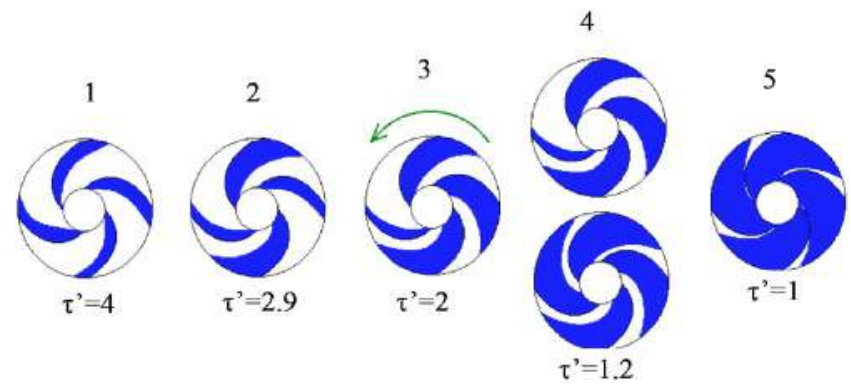

Fig. 2 Sketches of cavitation patterns for various cavitation parameters and their correspondence to the performance curve [1]

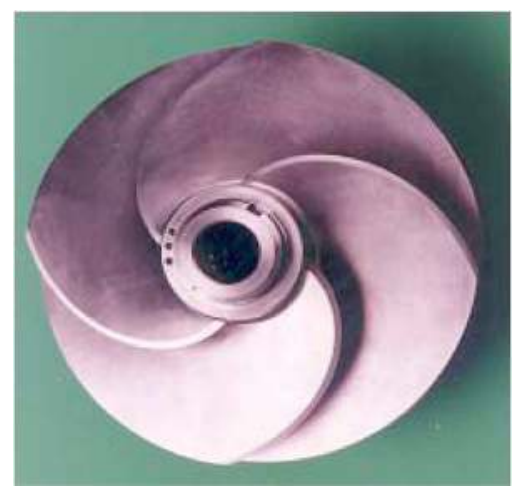

Fig. 3 3D inducer

the associated curvature of the blades. In these conditions, the inlet backflow is not relevant and a 2D geometry approach can be adopted for qualitative and parametric studies. It is important to note that tip clearance and backflow effects are neglected, and consequently, the applicability of the approach proposed in this paper might be limited to high inducer flow rates.

Hence, to study the unsteady behavior of the cavitating flow, some computations are performed in a four-blade cascade derived from an entire inducer. The transformation from the 3D geometry to the $2 \mathrm{D}$ blade cascade leads to neglect the peripheral cavitation in the inducer: only attached sheets of cavitation on the blades will be considered. To obtain the 2D geometry, the 3D inducer (Fig. 3) is cut at a constant radius $R_{c}$.

In the present paper we present results obtained for $R_{c}=70 \%$ of the tip radius $R$. The resulting shape of the blade cut in the plane $\left(R_{c} \cdot \theta, Z\right)$ is given in Fig. 4.

We use a $195 \times 40$ structured mesh per channel, giving a total of 31,200 internal nodes when calculating the four-blade cascade. A special contraction of the mesh is applied close to the blades (Fig. 5) to ensure that the first cells remain in the logarithmic region of the turbulent boundary layer, and apply the $k-\varepsilon$ RNG turbulence model with wall functions. The application of periodicity and connection boundary conditions needs also adequate meshes (small curvature and limited variation of cell sizes) in the connection zones between channels.

The calculations are done in the rotating frame. The imposed flow velocity $\mathbf{W}$ at the mesh inlet is given by

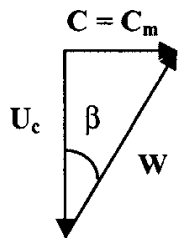

where

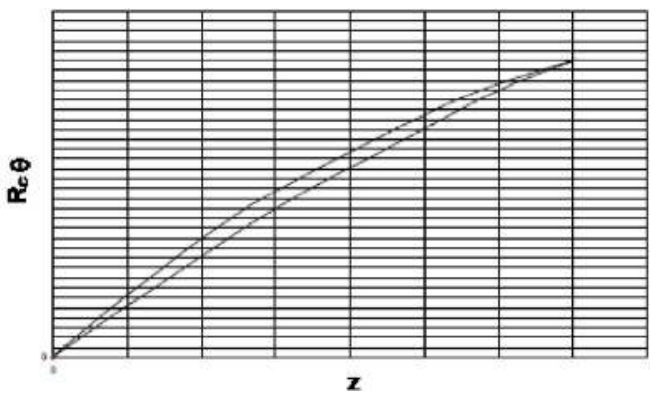

Fig. 4 Blade cut in the plane $\left(\boldsymbol{R}_{c} . \theta, Z\right)$ 

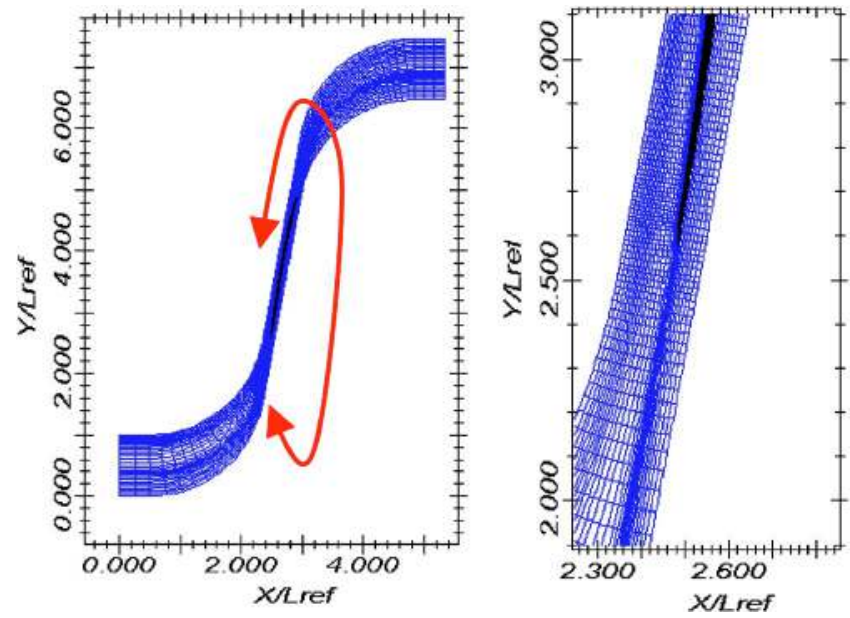

Fig. 5 Curvilinear, orthogonal mesh of a single channel (general view and zoom at the blade leading edge)

$$
\phi=\tan \beta=\frac{C_{m}}{\left(\Omega R_{C}\right)}
$$

The considered number Reynolds is equal to $10^{6}$ and the barotropic law slope is given by $A_{\min } / V_{\text {ref }}=0.1$. The convergence criterion is given by a residue of $10^{-6}$ for the mass balance equation and a residue of $3 \cdot 10^{-4}$ for momentum equations.

\section{Results}

Quasi-steady calculations are performed on the one-channel mesh and unsteady computations are performed on the four-blade mesh. The periodicity condition is then applied between the fourth and first channels, reflecting the true periodicity of the actual flow field. The time step, mesh, and turbulence model are chosen with the focus on capturing the low-frequency fluctuations of the attached cavity, rather than the local unsteadiness in the cavitation sheet wake (cloud shedding): the time step is fixed equal to $1 \%$ of the blade passage time $T_{\text {ref. }}$. Successive time accurate computations are performed at fixed cavitation number and flow-rate coefficient.

3.1 Head Drop Chart. Several four-channel computations are performed at nominal flow rate, with a cavitation number $\sigma$ varying from low-cavitation conditions down to the final perfor- mance drop. The corresponding head drop chart is drawn on Fig. 6. Some difference with the performance chart obtained with a single channel computation appears clearly, particularly for 0.6 $<\sigma<0.75$. This is due to the appearance, at these operating points, of unstable cavitating behaviors. We first present in Sec. 3.2 the results obtained in stable configurations $(\sigma>0.75$ or $\sigma$ $<0.6$ ), and then the mechanisms of unstable configurations will be detailed in Sec. 3.3.

3.2 Stable Configurations. At nominal flow rate, stable configurations correspond to cavitation numbers higher than 0.75 or lower than 0.6. Between these two extrema, unstable conditions are obtained, which will be detailed in Sec. 3.3.

For $\sigma=0.8$, sheets of cavitation are small, as indicated on Fig. $7(a)$. The four cavities remain identical, even if the calculation is continued for a long time, or if perturbations are simulated to enhance a possible instability. The time evolution of the cavity in the first channel, reported in Fig. 7(b), confirms that sheets of cavitation are completely stable after the initial transient. For these flow conditions, the performance of the inducer is only slightly affected by the presence of vapor, since its decrease does not exceed $5 \%$.

A similar configuration is obtained for a cavitation number equal to 0.6 (i.e., the inferior limit value). The sheets of cavitation are much more developed and responsible for the important performance drop that is observed at this operating point: the obstruction due to the cavities modifies the velocity fields on the blades, which leads to the head drop.

3.3 Unstable Configurations. For some particular values of the cavitation number, nonsymmetrical configurations of sheets of cavitation on the four blades are obtained. These configurations are periodical and involve physical fluctuations of almost constant magnitude in the flow field, after the initial transient period. Two main configurations were observed.

3.3.1 First Configuration for $\sigma=0.7$ (Nominal Flow Rate). At this operating point, mass flow rates in the four blade-to-blade channels become different. Their evolution is reported in Fig. 8. It appears that at a given time, the mass flow rate in channels 1 and 3 is very different from the mass flow rate in channels 2 and 4 . All evolutions are periodical and suggest a coupling between, respectively, channels 1 and 3, and channels 2 and 4. The corresponding evolution of the four sheets of cavitation between $T / T_{\text {ref }}=115$ and $T / T_{\text {ref }}=135$ is represented in Fig. 9.

It is clear in Fig. 9 that mass flow-rate variations in the different channels are strongly correlated with the size evolution of the

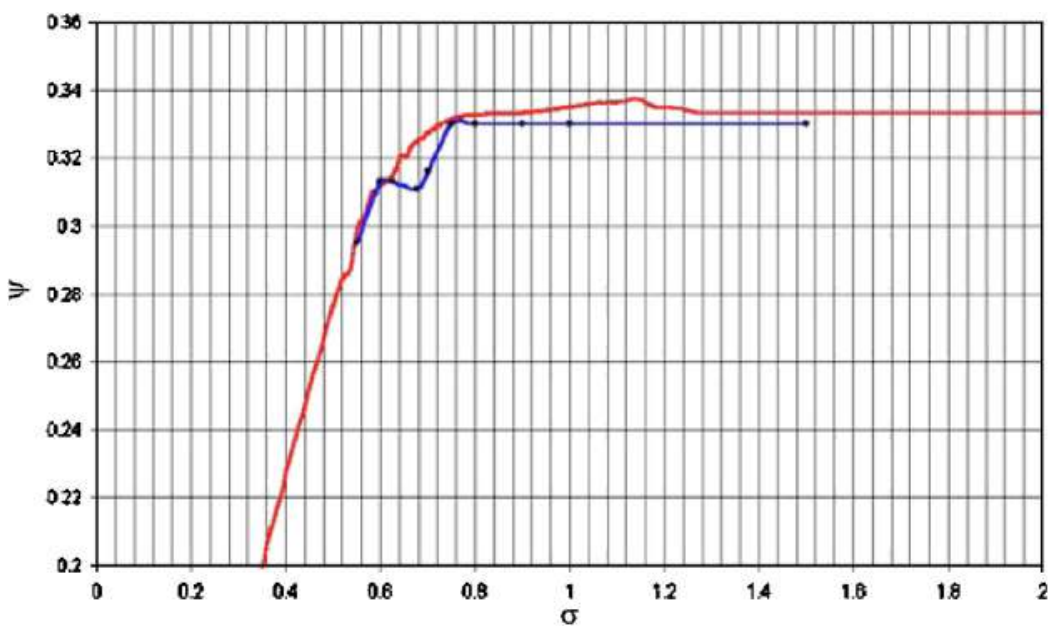

Fig. 6 Comparison between the performance charts obtained with four-blade computations (- - ) and with single blade computations (- - ) at nominal flow rate 

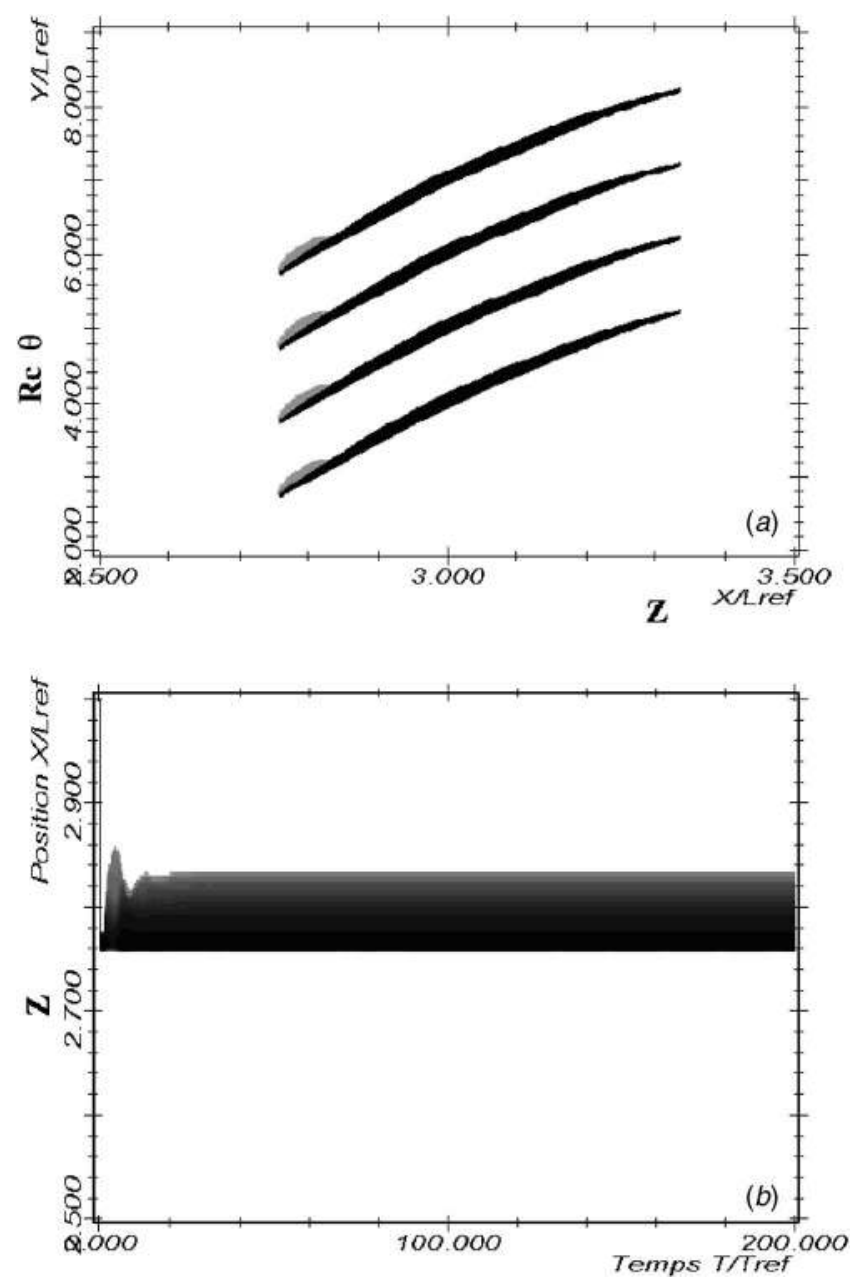

Fig. 7 Cavitation development for $\sigma=0.8$ and $Q=Q_{n}$ : (a) Density fields: white corresponds to pure liquid, and black to pure vapor (scale ratio $x / y \approx 10$ ). (b) Time evolution of the cavity in the first channel. The time is reported in abscissa, and the $Z$ position on the blade is graduated in ordinate. The colors (shading) represent the density values. At a given point in time and position, the color (shading) indicates the minimum density in the corresponding cross section of the channel.

sheets of cavitation. This continuous variation of cavities in channels 1 and 3 is the opposite of the one in channels 2 and 4, as for the mass flow rate in Fig. 8. In the first two configurations of Fig. 9 , for example, sheets of cavitation are growing in channels 1 and

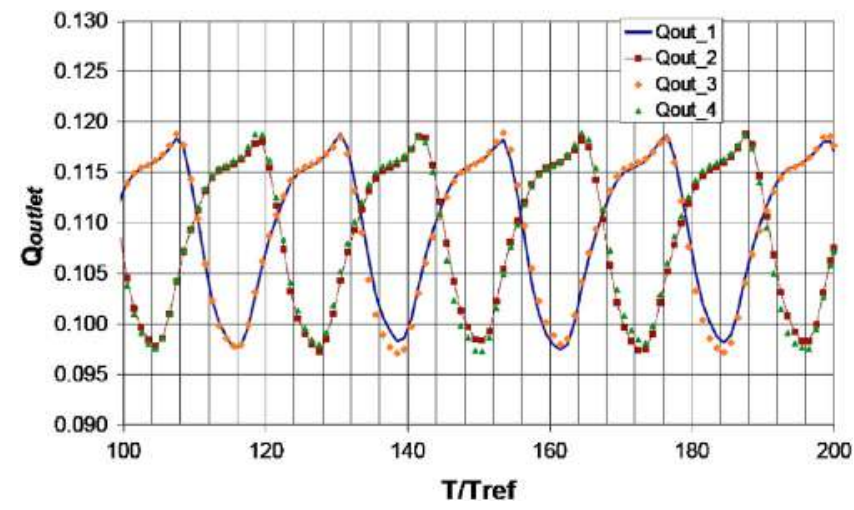

Fig. 8 Time evolution of the nondimension mass flow rate in the four channels $\left(\sigma=0.7\right.$ and $\left.Q=Q_{n}\right)$
3, whereas they decrease in channels 2 and 4; at the same time, mass flow rate is decreasing in channels 1 and 3, and increasing in channels 2 and 4 . This is due to the obstruction generated by the biggest cavities, which leads to a deviation of the flow toward free channels (i.e., channels in which cavities are small). This mechanism is amplified by the increase of the void ratio in the cavitation sheets during the step of growth: the vapor makes the flow to curve round the leading edge of the corresponding blades, and a part of the flow rate passes in the upper channel. This is illustrated by Fig. 10, which shows (inside the circle) the deviation imposed by the biggest cavities to the flow.

A spectral analysis of the mass flow-rate evolution downstream from the blades is proposed in Fig. 11(a). The frequencies are indicated in the rotating frame. A characteristic frequency $f_{c}$ $=0.16 f_{0}\left(f_{0}\right.$ is the rotating frequency of the inducer $)$ is obtained, which is consistent with the periodical mass flow-rate evolutions reported previously in Fig. 8. Indeed, $f_{c}$ corresponds to the evolution of the sheets of cavitation on the blades. This phenomenon also appears on the spectral analysis in the absolute frame of the static pressure far upstream from the blades, drawn in Fig. 11(b): the frequency $f_{c a}=0.32 f_{0}$ is predominant. $f_{c a}=2 \times f_{c}$, the factor 2 being due to the alternate cavitation pattern on the blades. We can observe also other frequencies of about $4 f_{c}, 6 f_{c}$, and $8 f_{c}$.

In order to deepen the frequency study, we have plotted also the spectral analysis of the total pressure signal near to the leading edge of the blade cascade in the absolute frame (Fig. 12). These results indicate two characteristic frequencies of $\left(2 f_{0}+f_{c}\right)$ and $\left(2 f_{0}-f_{c}\right)$. The combination of these frequencies reproduces mass flow fluctuation with the frequency of $f_{c}$ in the rotating frame shown in the Fig. 11(a). Moreover, the combination of these frequencies seems to be also related to the frequencies $2 f_{c}, 4 f_{c}, 6 f_{c}$, and $8 f_{c}$ observed in Fig. 11(b).

We will name this configuration "unstable alternate cavitation." This special configuration was found only for the values $(\sigma=0.7$, $\left.Q_{n}\right)$ and $\left(A_{\min } / V_{\text {ref }}=0.1, \operatorname{Re}_{y}=10^{6}, 10<y^{+}<60\right)$. To deepen the analysis of this configuration, we have performed numerical tests with different meshes, Reynolds numbers, $y^{+}$and time-step values. According to our numerical tests, for a different mesh $(390$ $\times 60 ; 10<y^{+}<20 ; A_{\min } / V_{\text {ref }}=0.1$, Rey $\left.=10^{6}\right)$, the simulations give a regular super synchronous cavitation instead of unsteady alternate cavitation.

For this operating point $\left(\sigma=0.7, Q_{n}\right)$ and $195 \times 40$ mesh, the numerical tests pointed out also the influence of the following:

- The celerity value: for $A_{\min } / V_{\text {ref }}=0.04$ (Rey $=10^{6}, 10<y^{+}$ $<60$ ), we observe a regular supersynchronous cavitation with a characteristic frequency of $1.6 f_{0}$. As a matter of fact, for smaller $A_{\min }$ values, the cavitation length and thickness change and the unsteady cavitation behavior is also modified.

- The Reynolds number: for Rey $=3.10^{6}$ (corresponding to a range of $20<y^{+}<100$ in the case of $195 \times 40 \mathrm{mesh}$ ), the unsteady alternate cavitation becomes very irregular and changes to a supersynchronous configuration. Indeed, the trigger mechanism of the instabilities seems to be related to the friction in the boundary layer flow and, consequently, to the values of $y^{+}$(calculated as a function of Reynolds number and mesh thickness).

It is interesting to note that a recent work [17], concerning the same geometry but based on a different numerical model and mesh $\left(20<y^{+}<100\right)$, pointed out also an unsteady alternate cavitation configuration for $\sigma=0.7$ and $Q=Q_{n}$. In this same work, a steady alternate cavitation was observed for $\sigma$ of $\sim 0.77$.

3.3.2 Second Configuration for $\sigma=0.625$ (Nominal Flow Rate). If the cavitation number is decreased down to a value between 0.6 and 0.65 , another type of unstable behavior is obtained. It is characterised by four different sizes of the sheet of cavitation 


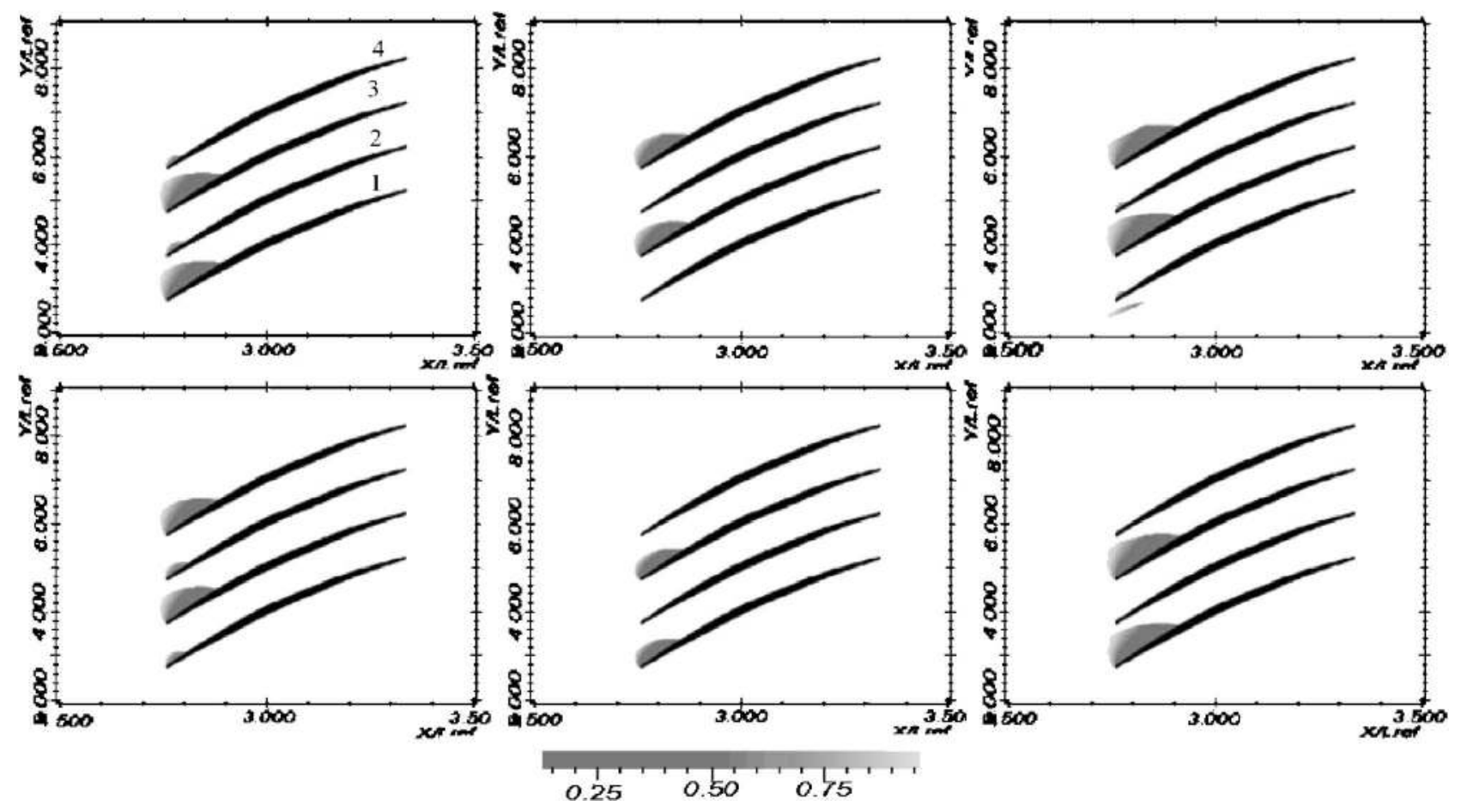

Fig. 9 Time evolution of the sheets of cavitation $\left(110<T / T_{\text {ref }}<135\right)$

in the four channels. No simple correlation between channels is detectable at any time, as can be seen in Fig. 13, in which the evolution during $155 \mathrm{~T} / T_{\text {ref }}$ of the four mass flow rates in the four channels is reported. As previously, the instability is periodical after the initial transient period, with mass flow rate fluctuations of constant magnitude in all channels. Nevertheless, the mechanisms that govern the cavity evolution in each channel are much more complicated than in the first unstable configuration, since all cavities interact.

The spectral analysis of the mass flow rate downstream from the blade is proposed in Fig. 14(a). A characteristic frequency $f_{c}$ $=0.38 f_{0}$ clearly appears in the rotating frame, with two harmonic frequencies. It corresponds to the evolution of the mass flow rates in the four channels, and this phenomenon in the absolute frame has a higher frequency than the inducer rotation. The main char-

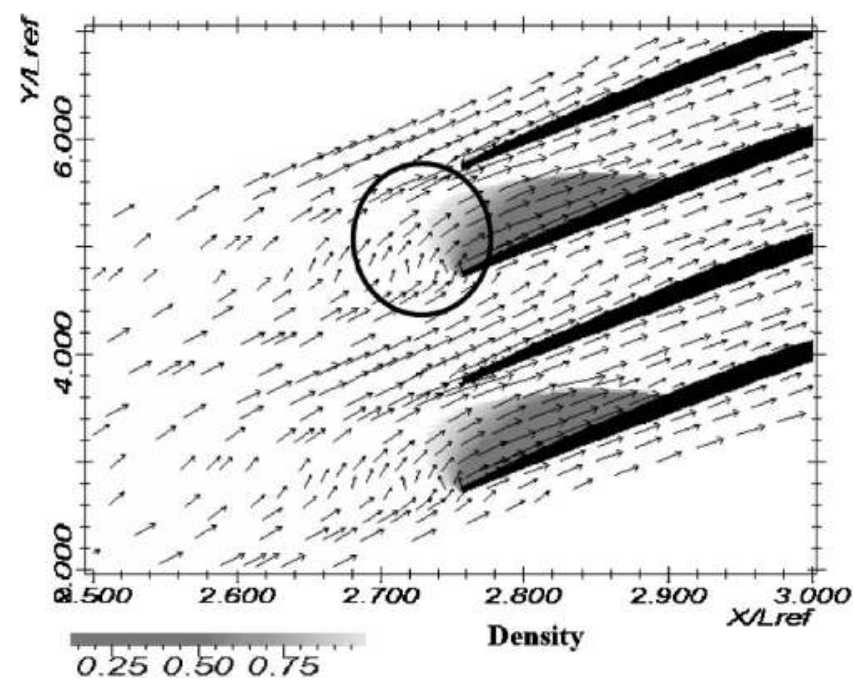

Fig. 10 Deviation of velocities due to high void ratio ( $T=110$ $\left.T_{\text {ref }}\right)$ acteristic frequency $f_{c}=1.38$ in the absolute frame is observed, from the spectral analysis of the upstream static pressure evolution drawn in Fig. 14(b). This is the reason why such unstable configuration is called supersynchronous rotating cavitation. It means that the configuration in the inducer at a given time (the four different sizes of the cavities) rotates faster than the inducer
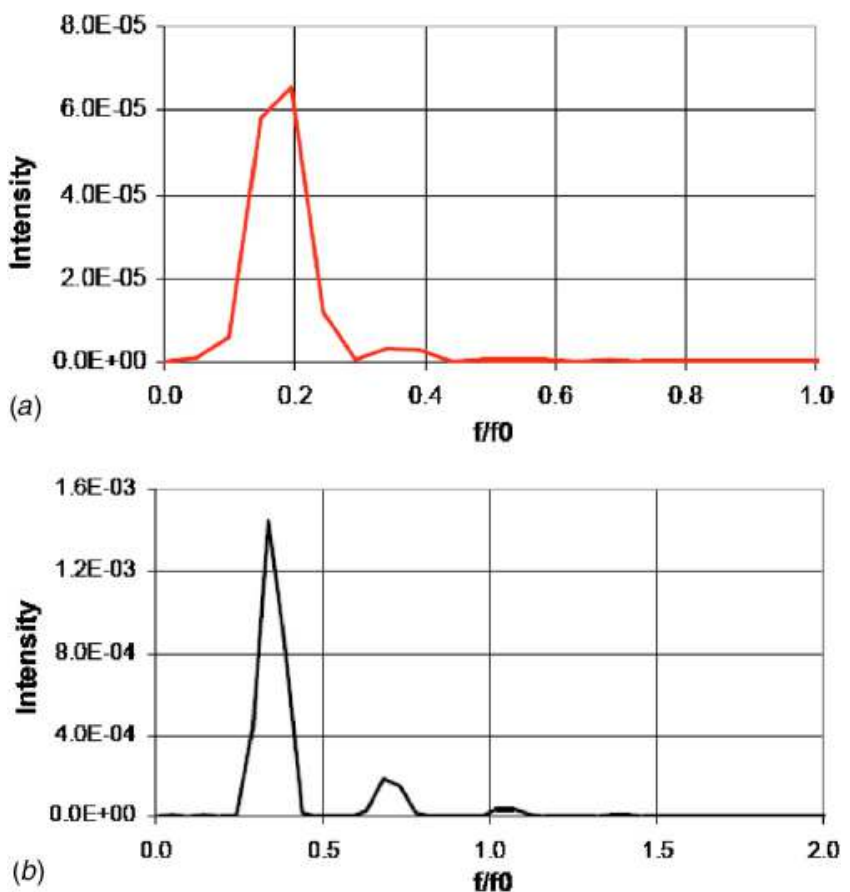

Fig. 11 Spectral analysis $\left(\sigma=0.7, Q=Q_{n}\right)$ : (a) Mass flow rate downstream from the blades, in the rotating frame and $(b)$ static pressure far upstream from the blades, in the absolute frame (results concerning a calculation time of about 30 rotation periods of the inducer) 

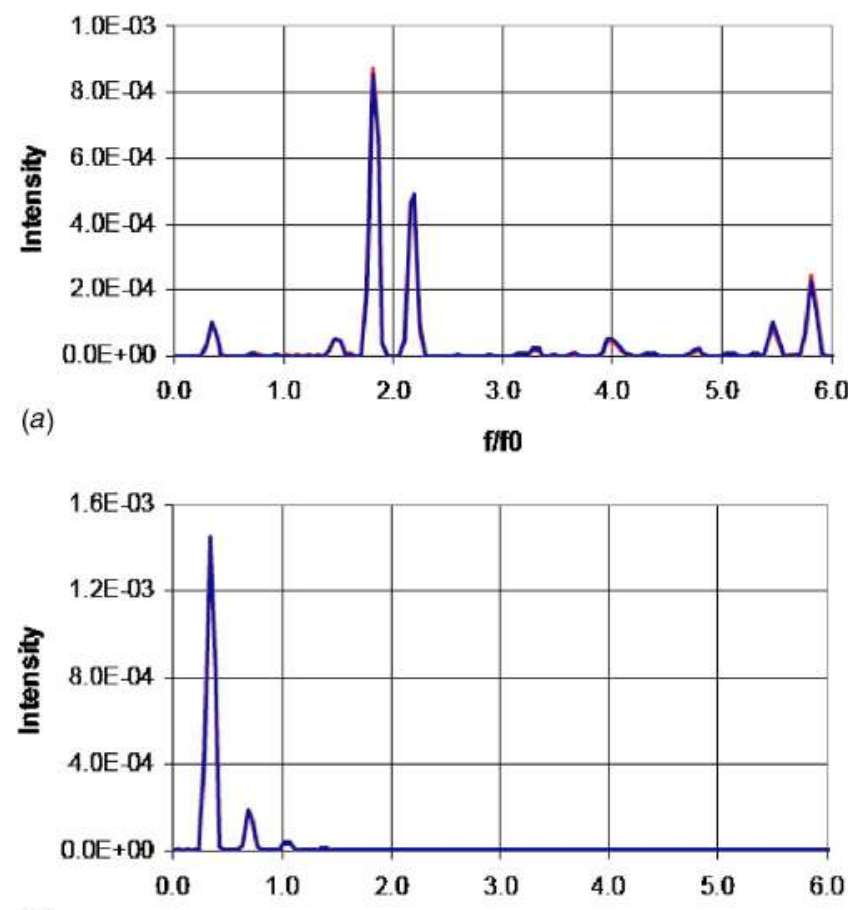

(b)

f:F

Fig. 12 Spectral analysis $\left(\sigma=0.7, Q=Q_{n}\right)$ : (a) Total pressure near to the leading edge, in the absolute frame and $(b)$ total pressure far upstream from the blades, in the absolute frame (results concerning a calculation time of $\sim 30$ rotation periods of the inducer)

itself.

All frequency analyses performed in the present study have been carried out from calculations whose duration was at least 25 periods of inducer rotation. The whole of performed unsteady cavitating simulations represent a great effort in terms of computation time, and, at present, these kinds of frequency analyses cannot be done from 3D calculations. It is worth noting that the considered time step and time calculations do not allow us to detect very low frequencies (below $0.1 f_{0}$ ).
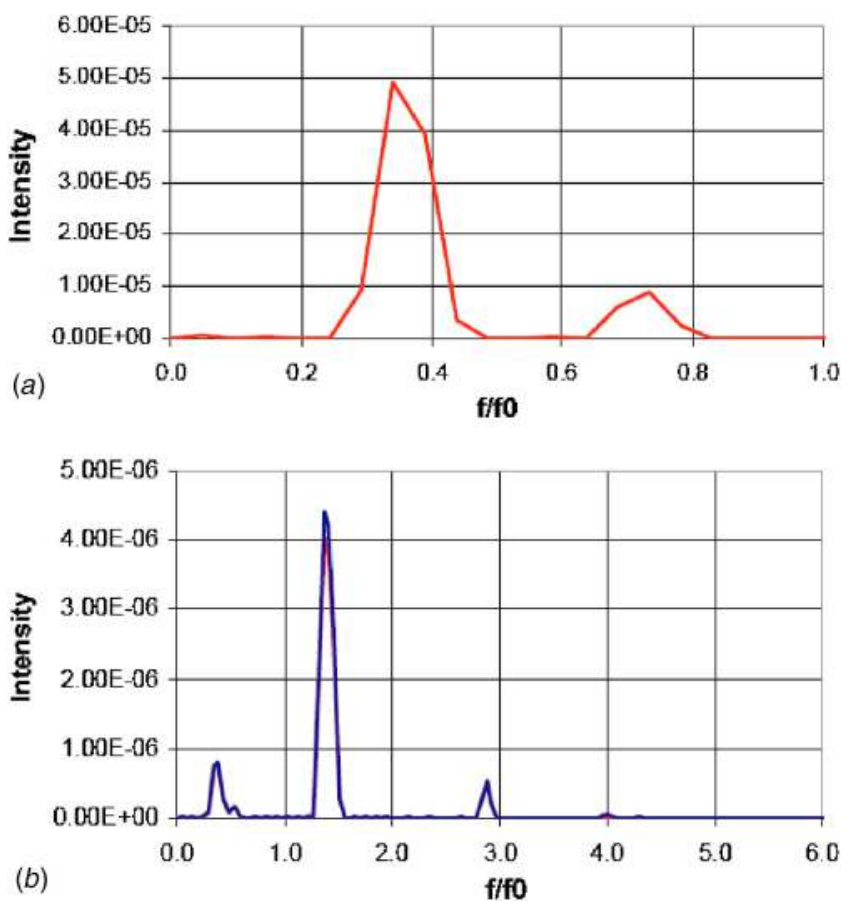

Fig. 14 Spectral analysis $\left(\sigma=0.625, Q=Q_{n}\right)$ : (a) Mass flow rate downstream from the blades, in the rotating frame and $(b)$ static pressure far upstream from the blades, in the absolute frame (results concerning a calculation time of $\sim 30$ rotation periods of the inducer)

\subsection{Efforts on the Blade in Unstable Configurations.}

3.4.1 Unstable Alternate Cavitation. To improve the understanding of the effects of cavitation instabilities on the blade and on the entire inducer, the fluctuations of the forces acting on the blades have been calculated and drawn in Fig. 15. The objective is to estimate the magnitude of the stress fluctuations. The stresses are calculated through the momentum conservation in a control volume surrounding one blade. Projecting this relation, respectively, onto the normal to the inlet boundary and onto the normal to the rotation axis gives the axial and the transverse components of the nondimensional hydrodynamic force, whose time evolution

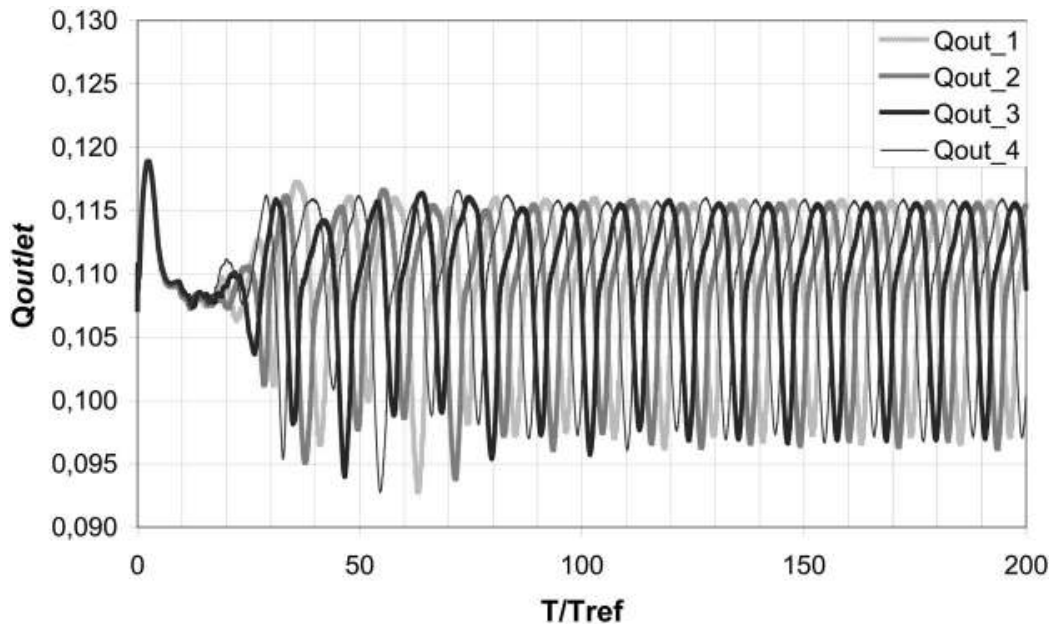

Fig. 13 Time evolution of the non dimension mass flow rates in the four channels $\left(\sigma=0.625, Q=Q_{n}\right)$ 

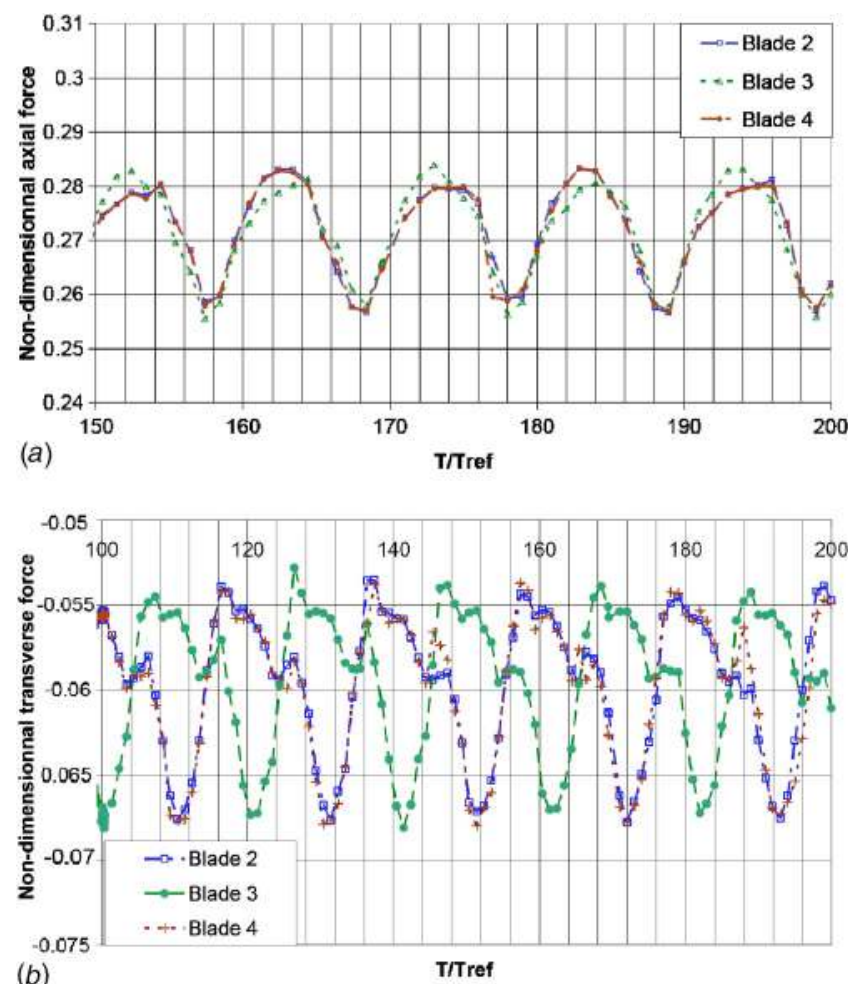

Fig. 15 Time distribution of the axial and transverse forces on the blades $\left(\sigma=0.7\right.$ and $\left.Q=Q_{n}\right)$

is then represented in Figs. 15(a) and 15(b) for three different blades. Stress fluctuations in these figures are generated by unsteady cavitation.

The mean nondimensional axial force $F_{x} /\left(\rho_{\text {ref }} V_{\text {ref }}^{2} L_{\text {ref }}^{2}\right)$ can be estimated to 0.27 , whatever blade we consider, and the order of magnitude of the fluctuations is $\sim 0.016$. Thus, cavitation instabilities lead in the present case to fluctuations of the axial force equal to $6 \%$ of the mean force. In the case of the transverse force, the fluctuations reach $13 \%$ of the mean force.

3.4.2 Supersynchronous Configuration. The corresponding force evolutions are represented in Figs. 16(a) and 16(b). In the case of the second blade, for example, the mean axial force still equals 0.27 , while the fluctuations magnitude is $\sim 0.03$ : rotating cavitation is responsible, in this case, for fluctuations whose magnitude can be estimated to $10 \%$ of the mean effort. In the case of the transverse force, the fluctuations reach almost $20 \%$ of the mean force.

According to these first calculations, the simulated unstable alternate cavitation seems to be less critical for the inducer than with the calculated supersynchronous rotating cavitation. In this case, axial forces on the blades substantially increase and the periodical forces acting on the blades could be responsible for some damage to the inducer.

\section{Conclusion}

Computations were performed at different operating conditions by varying the mass flow rate and the cavitation number. The resulting performance charts of the blade cascade at the calculated mass flow rates are reported in Fig. 17. Stable and unstable cavitation configurations are also indicated, so that the limit of the rotating cavitation behavior can be identified. Results for mass flow rates $<0.9 Q_{n}$ are not reported because of the numerical difficulties that we encountered at these flow conditions. Indeed, at partial flow rate, angle of attack of the flow at the blade leading edge and cavity thickness both increase. In case of rotating behav-
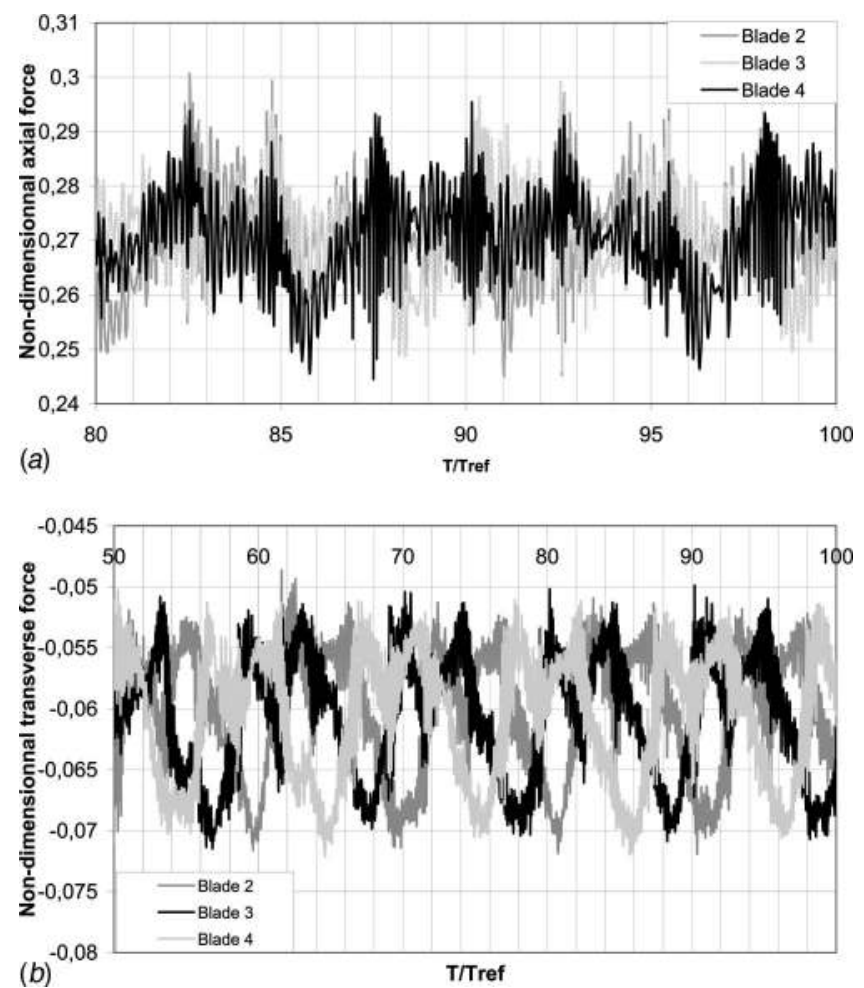

Fig. 16 Axial and transverse forces on the blades $(\sigma=0.625$ and $\left.Q=Q_{n}\right)$

ior, it can result in the complete obstruction of a channel, which induces serious numerical problems. Moreover, at partial flow rates, tip leakage and backflow influence cannot be neglected and a 2D approach would probably lead to high errors.

It can be seen (Fig. 17) that the cavitation parameter range of rotating cavitation increases when the flow-rate coefficient decreases. This result agrees with experimental observations $[3,18]$. At nominal flow rate $Q_{n}$, the experimental instability range is about $\Delta \sigma_{\text {exp. }}=0.15$, which is consistent with the results obtained previously by [16] in the case of a similar four-blade inducer geometry. The mass flow-rate limit for appearance of unstable configuration $\left(1.2 Q_{n}\right)$ is also identical to the one obtained in this previous study. Further work is now needed to assess the prediction capability of the model. Therefore, improvements of the analysis of 3D inlet flows and study of the influence of the cascade design by numerical simulations are in progress, in parallel to the development of a full 3D model $[17,19]$.

\section{Acknowledgment}

The authors wish to thank Snecma (Rocket Engine Division) and the French space agency CNES for their support to the present work.

\section{Nomenclature}

$A_{\text {min }}=$ slope of the barotropic state law: $A_{\min }^{2}=\partial P / \partial \rho$ (minimum speed of sound in the vapor/liquid mixture)

$Q, Q_{n}=$ nondimensional mass flow rate, nominal mass flow rate

$\mathbf{C}\left(\mathrm{C}_{\mathrm{m}}, \mathrm{C}_{\mathrm{u}}\right)=$ velocity vector in fixed frame: $C_{\mathrm{m}}=Q /\left(\rho S_{\text {flow }}\right)$

$L_{\text {ref }}=$ reference length $L_{\text {ref }}=\pi R_{\mathrm{c}} / 2$

$p, P=$ static pressure, total pressure: $P=p+0.5 \rho_{\mathrm{l}} \mathbf{C}^{2}$

$p_{v}=$ vapor pressure

$R_{c}=$ cut radius for the passage from $3 \mathrm{D}$ to $2 \mathrm{D}$

$R, \theta, Z=$ cylindrical coordinates 


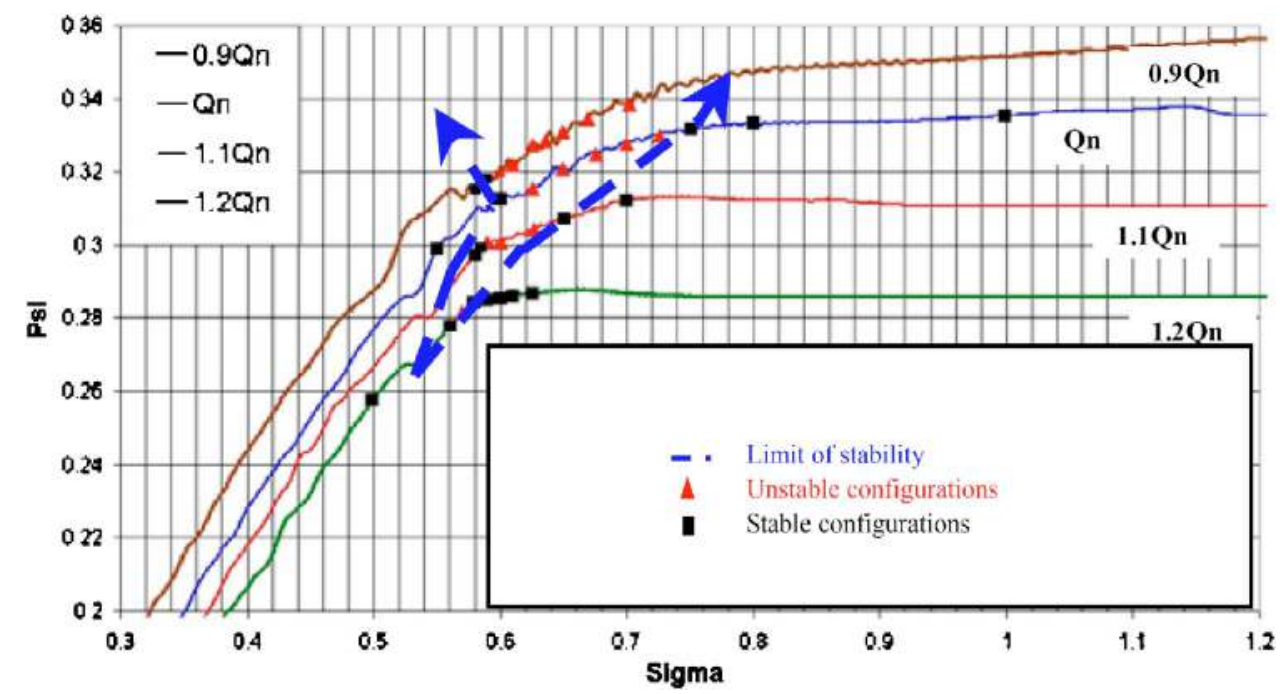

Fig. 17 Performance charts at several mass flow rates

$T_{\text {ref }}=$ reference time: $T_{\text {ref }}=L_{\text {ref }} / V_{\text {ref }}$

$V_{\text {ref }}=$ reference velocity: $V_{\text {ref }}=R_{c} \Omega$

$\mathbf{W}\left(W_{\mathrm{m}}, W_{\mathrm{u}}\right)=$ velocity vector in relative frame

$y^{+}=$nondimensional distance to the boundary

$\Omega=$ angular rotation speed of the inducer $(\mathrm{rad} / \mathrm{s})$

$U=$ training velocity at inducer tip radius $R_{\text {tip }}: U$ $=R_{\text {tip }} \Omega$

$\tau, \tau^{\prime}=$ cavitation parameter for inducers, $\tau^{\prime}=\tau / \tau_{c}$

$\tau_{c}=$ critical value. $\tau=\left(P_{\text {inlet }}-p_{v}\right) /\left(0.5 \rho_{1} R_{\text {tip }}^{2} \Omega^{2}\right)$

$\sigma=$ cavitation nb (downstream): $\sigma=\left(P_{\text {outlet }}-p_{\mathrm{v}}\right) /\left(0.5 \rho_{1} V_{\text {ref }}^{2}\right)$

$f, f_{0}=$ frequency, inducer rotation frequency

$\Psi=$ head coefficient $\left(P_{\text {outlet }}-P_{\text {inlet }}\right) /\left(\rho_{1} R_{\text {tip }}^{2} \Omega^{2}\right)$

$\rho_{\mathrm{l}}, \rho_{\mathrm{v}}, \rho=$ specific mass of the liquid, of the vapor, of the mixture

$\alpha=$ local void ratio: $\alpha=\left(\rho-\rho_{\mathrm{l}}\right) /\left(\rho_{\mathrm{v}}-\rho_{\mathrm{l}}\right)$

\section{References}

[1] de Bernardi, J., Joussellin, F., and Von Kaenel, A., 1993, "Experimental Analysis of Instabilities Related to Cavitation in Turbopump Inducer," 1st Int. Symp. on Pump Noise and Vibrations, Paris, France, pp. 91-99.

[2] Kamijo, K., Shimura, T., and Watanabe, M., 1977, "An Experimental Investigation of Cavitating Inducer Instability," ASME Paper No. 77-WA/FW-14.

[3] Pagnier, P., Morel, P., Spettel, F., Henry, C., and Champagne, J.-Y., 1995, "Conception and Experimental Study of an Inducer," Cav'95 Int. Symp., Deauville, France.

[4] Goirand, B., Mertz, A., Joussellin, F., and Rebattet, C., 1992, "Experimental Investigation of Radial Loads Induced by Partial Cavitation with Liquid Hydrogen Inducer," 3rd Int. Conf. on Cavitation, IMechE, C453/056, Cambridge, England, pp. 263-269.

[5] Tsujimoto, Y., 2001, "Simple Rules for Cavitation Instabilities in Turbomachinery," 4th Int. Symp. on Cavitation, Pasadena.

[6] Tsujimoto, Y., Kamijo, K., and Yoshida, Y., 1993, “A Theoretical Analysis of Rotating Cavitation Inducers,” ASME J. Fluids Eng., 115, pp. 135-141.
[7] Joussellin, F., and de Bernardi, J., 1994, "Analytical Modelling of Instabilities in a Cavitating Turbopump Inducer," 2nd Int. Symp. on Cavitation, Tokyo, pp. 98-94.

[8] Horiguchi, H., Watanabe, S., Tsujimoto, Y., and Aoki, M., 2000, "Theoretical Analysis of Alternate Blade Cavitation in Inducers," ASME J. Fluids Eng., 122(1), pp. 156-163.

[9] Horiguchi, H., Watanabe, S., and Tsujimoto, Y., 2000, "A Linear Stability Analysis of Cavitation in a Finite Blade Count Impeller," ASME J. Fluids Eng., 122(4), pp. 798-805.

[10] Pilipenko, V., Semyonov, Y., and Kvasha, Y., 1995, "Theoretical and Experimental Computational Methods for Determining Volume of Cavities and Coefficients of Dynamic Equation of Cavitation Cavities," Tech. Report No. SEP TC/T 23227/96.

[11] Watanabe, S., Tsujimoto, Y., Franc, J.-P., and Michel, J.-M., 1998, "Linear Analyses of Cavitation Instabilities," 3rd Int. Symp. on Cavitation, Grenoble, pp. 347-352.

[12] Watanabe, S., Sato, K., Tsujimoto, Y., and Kamijo, K., 1999, "Analysis of Rotating Cavitation in a Finite Pitch Cascade Using a Closed Cavity Model and a Singularity Method," ASME J. Fluids Eng., 121(4), pp. 834-840.

[13] Delannoy, Y., and Kueny, J. L., 1990, "Two Phase Flow Approach in Unsteady Cavitation Modelling," Cavitation and Multiphase Flow Forum, ASME-FED Vol. 98, pp. 153-158.

[14] Reboud, J. L., and Delannoy, Y., 1994, "Two Phase Flow Modelling of Unsteady Cavitation," 2nd Int. Symp. on Cavitation, Tokyo, pp. 39-44.

[15] Coutier-Delgosha, O., Reboud, J.-L., and Delannoy, Y., 2003, "Numerica Simulation of the Unsteady Behaviour of Cavitating Flows," Int. J. Numer. Methods Fluids, 42, pp. 527-548.

[16] Coutier-Delgosha, O., Courtot, Y., Joussellin, F., and Reboud, J.L., 2004, “Numerical Simulation of the Unsteady Cavitation Behavior of an Inducer Blade Cascade," AIAA J., 42(3), pp. 560-569.

[17] Pouffary, B., Fortes-Patella, R., and Reboud, J. L., 2005, "Numerical Analysis of Cavitation Instabilities in Inducer Blade Cascade," 5th Int. Symp. on Pumping Machinery, ASME 2005 Summer Meeting, Houston.

[18] Yokata, K., Kurahara, K., Kataoka, D., Tsujimoto, Y., and Acosta, A. J., 1999, "A Study on Swirling Backflow and Vortex Structures at the Inlet of Inducer," JSME Int. J., Ser. B, 42-3, pp. 451-459.

[19] Pouffary, B., Fortes-Patella, R., and Reboud, J. L., 2005, "Numerical Simulation of 3D Cavitating Flows: Analysis of Cavitation Head Drop in Turbomachinery," 5th Int. Symp. on Pumping Machinery, ASME 2005 Summer Meeting, Houston. 